\title{
A review of biological fluid power systems and their potential bionic applications
}

DOI:

10.1007/s42235-019-0031-6

\section{Document Version}

Accepted author manuscript

Link to publication record in Manchester Research Explorer

\section{Citation for published version (APA):}

Liu, C., Wang, Y., Ren, L., \& Ren, L. (2019). A review of biological fluid power systems and their potential bionic applications. Journal of Bionic Engineering. https://doi.org/10.1007/s42235-019-0031-6

\section{Published in:}

Journal of Bionic Engineering

\section{Citing this paper}

Please note that where the full-text provided on Manchester Research Explorer is the Author Accepted Manuscript or Proof version this may differ from the final Published version. If citing, it is advised that you check and use the publisher's definitive version.

\section{General rights}

Copyright and moral rights for the publications made accessible in the Research Explorer are retained by the authors and/or other copyright owners and it is a condition of accessing publications that users recognise and abide by the legal requirements associated with these rights.

\section{Takedown policy}

If you believe that this document breaches copyright please refer to the University of Manchester's Takedown Procedures [http://man.ac.uk/04Y6Bo] or contact uml.scholarlycommunications@manchester.ac.uk providing relevant details, so we can investigate your claim.

\section{OPEN ACCESS}




\title{
A review of biological fluid power systems and their potential bionic
}

\section{applications}

\author{
Chunbao Liu ${ }^{1,2}$, Yingjie Wang ${ }^{1,2}$, Luquan Ren ${ }^{2}$, Lei Ren ${ }^{2,3 \Uparrow}$ \\ 1 School of Mechanical and Aerospace Engineering, Jilin University, Changchun 130022, China \\ 2 Key Laboratory of Bionic Engineering, Ministry of Education, Jilin University, Changchun
}

130022, China

3 School of Mechanical, Aerospace and Civil Engineering, University of Manchester, Manchester

M13 9PL, UK

\begin{abstract}
Nature has always inspired human achievements in industry, and biomimetics is increasingly being applied in fluid power technology. Arachnida use hydraulic forces, rather than muscle, for leg extensions during locomotion. Many cold-blooded and soft-bodied organisms rely on a hydrostatic skeleton to transmit force, which involves a hydraulic mechanism. Biological hydraulic transmission differs from engineering hydraulic transmission in many aspects, such as in energy transfer and transformation, the movement mode, environmental friendliness, system pressure level, and energy supplement mode. The existence of a hydraulic mechanism in a biological drive requires 3 features: a power source, cavity, and working medium. The power source is similar to a hydraulic pump, and the cavity is similar to a hydraulic cylinder, both of which are necessary for producing deformation. The working medium is similar to a hydraulic fluid. Under these 3 conditions, a biological flow is generated inside or outside the body to meet the needs of a biological drive. This paper reviews the biological organisms that employ hydraulic systems, identifies related studies on these biological hydraulic systems, and summarizes the mechanisms involved in using hydraulic pressure to achieve graceful and agile movements. This in-depth study and exploration of biological hydraulic systems can provide a good reference for solving the challenges of using hydraulic systems, such as increasing the energy efficiency, improving reliability, building smart components and systems, reducing the size and weight of components, reducing the environmental impact of systems, and improving and applying energy storage and redeployment capabilities. This paper also includes a detailed discussion of new ideas and innovative sources for the future development of hydraulic systems. In contrast with the bio-inspired designs used in other engineering fields, very few studies have reported on using bio-inspired methods for hydraulic transmission techniques. The aim of this work is to attract the attention of researchers to help address this gap and to promote the use of biologically-inspired methods to improve engineering fluid power systems.
\end{abstract}

Keywords: Bionic; Fluid power; Pressurized liquids; Locomotion; Bio-inspired applications

\section{Introduction}

Can you imagine the links from cell division and self-defense in the deep ocean to the rapid locomotion of arthropods? The relevant mechanism can be described simply as the use of fluid

\footnotetext{
${ }^{\Uparrow}$ Corresponding author: lei.ren@manchester.ac.uk
} 
pressure changes in a sealed space to provide power transmission, which is in fact Pascal's law and the basis of fluid power. Fluid power provides a means of transmitting and controlling energy, and this power is transmitted primarily by increasing the liquid pressure energy. As hydraulic systems have the advantages of a high power-to-weight ratio, high force-to-mass ratio, rapid response, configurability, and self-lubrication, they dominate most engineering fields, either partially or totally ${ }^{11}$. Hydraulic transmission is an important industrial technology, there is an urgent need for hydraulic transmission systems that employ new materials and technologies that can be used in a broad range of applications $[2-5]$.

The circulatory system in humans is a remarkable hydraulic system; it includes a double pump delivering a fluid flow rate of approximately $10 \mathrm{~L} / \mathrm{min}$ at 0.16 bar maximum pressure and feeds a pipe network stretching more than 100,000 km. However, in engineering, locomotion must be more focused. Biological systems contain especially rich and useful information for a range of disciplines, such as locomotion systems ${ }^{[6]}$. Many organisms use pressurized liquid in vivo or in vitro to transmit power. For example, spiders use hydraulic force, rather than muscle, to extend the leg during locomotion [7]. Starfish use a hydraulic system called the water-vascular system (WVS) and tube feet to move ${ }^{[8]}$. Additionally, mollusks, bivalves, worms, and some insect larvae also utilize hydraulic transmission. All organisms compete with each other for the available energy. Hence, organisms' transmission systems arise from natural evolution and are adapted to integrate perfectly with the environment.

Engineered hydraulic transmission systems face numerous challenges, especially from the competition arising from motor drives in low-power applications. Therefore, it is necessary to determine how to combine new technologies, theories, and methods to improve the competitiveness of hydraulic transmission systems and expand their use into novel applications. Natural systems have unique advantages over engineered systems in terms of their intelligence, efficiency, weight, and environmental friendliness. Hence, these systems can serve as models for researchers, providing important inspiration to solve technical bottlenecks and contributing to the development of a creative new bionic hydraulic transmission system. The inspiration from nature has led to the development of effective materials, structures, tools, mechanisms, processes, algorithms, methods, and systems ${ }^{[9]}$. We reviewed studies related to biological hydraulic systems that were employed to accomplish locomotion in aquatic and terrestrial environments. We evaluated the range of the natural hydraulic diversity and established a connection with future engineering hydraulic systems that use robotic drive technology based on the existing levels and angles of structures, materials, seals, working media, drive principles, and energy conversion mechanisms. Combined with the shortcomings, challenges, and developing trends in engineering hydraulics, these connections may encourage more scientists and engineers to research biological hydraulic transmissions to discover new ideas and methods for solving hydraulic transmission problems and challenges. We hope to inspire solutions to practical engineering problems from natural systems.

\section{Fluid power in animals and engineering}

\subsection{Engineered hydraulic systems}

A hydraulic system is composed primarily of a hydraulic pump (power components), hydraulic cylinders/hydraulic motors (actuators), hydraulic valves (control components), fuel tanks/accumulators/filters/pipes, and heat exchangers (auxiliary components). A hydraulic system is primarily composed of a typical circuit. A sketch of the composition and the symbols for hydraulic 
components are shown in Fig. 1. Several types of energy conversion are necessary to achieve power transmission. An internal combustion engine has a power component. When activated, the mechanical energy generated by the internal combustion engine is applied to the hydraulic pump. Then, the mechanical energy is transformed into the fluid pressure energy, which is then transmitted to the actuator-hydraulic cylinder/hydraulic motor to complete a straight line/rotary motion. Then, the pressure energy is again converted into mechanical energy. A flow chart is shown in Fig. 2 to illustrate the process. Hydraulic valves are important for the development of hydraulic systems. They functionally adjust the system pressure, flow, direction, and other parameters to meet the needs of the components and provide motion. There are many different types of values, and the same valve may exhibit different functions when applied in different circuits. Electrical energy, in addition to providing a form of energy for hydraulic systems, was first applied to hydraulic valves. Later, the electric control valve emerged, followed by electrohydraulic servo-control systems, further expanding the applications of hydraulic systems.

Hydraulic power transmission has the advantages of a high-power density ratio, large output force and torque, desirable dynamic characteristics, flexible layout, and diverse control modes. This technology has been widely used in walking robots, such as Atlas ${ }^{[10]}$, HyQ $\left.{ }^{[11}, \underline{12}\right]$, HyQ2Max ${ }^{[13]}$, and lower limb exoskeletons [14, 15]. At present, the power density of hydraulic systems remains unmatched by electric drives; therefore, hydraulic robots can potentially function sufficiently well to surpass electric robots. However, there are challenges to the use of hydraulic systems. For example, the primary disadvantages of hydraulic transmission systems are their technical complexity and insufficient energy efficiency. Most hydraulic systems are energy inefficient, as low as $38.5 \%$, which is a considerable limitation to untethered operation time ${ }^{[16]}$.

Hydraulic systems were subject to a number of constraints during their development, including the need for high speeds and limitations created by friction materials, lubrication, and sealing. In mobile systems such as robots, the hydraulic system motor, pump, oil tank, and other components must all follow the movement of the host, which inevitably causes problems with the equipment. However, natural biological systems have overcome these issues. It is worth exploring whether nature can provide the inspiration for the development of hydraulic systems. Therefore, we have turned our attention to the graceful and agile movements of living systems that use hydraulic transmission in nature and have explored their composition, principles, and driving mechanisms.

\subsection{Biological hydraulic systems}

Organisms evolved hydraulic transmission systems gradually to primarily achieve the drive for their positive displacement pumps. Vogel explored the generalizations that emerge from considering an organism's pump fluids that have functional, phylogenetic, and structural diversity ${ }^{[17-22]}$. Bach et al. presented the current biomimetic research for state-of-the-art pumps and assessed their biomimetic potential for improving man-made pumping systems $[23]$.

Animals are composed almost entirely of soft materials and liquids ${ }^{[24]}$. Natural fluid power is linked to a highly integrated actuation system: the hydrostatic skeleton with a fluid-filled cavity (FFC). The FFC, also called the coelom or hemocoel, works as a hydraulic system, and organisms with an FFC have a very simple body structure that is mostly tubular. The fluids include blood, intracellular fluid, and seawater, depending on the animal taxon. Fluid-filled hydrostatic supports exhibit two forms of constant volume - that exemplified by the leech, which is sectional isochoric, and that exemplified by the caterpillar, which is whole-body isochoric [25]. To vary their body stiffness, invertebrates have a hydrostatic skeleton, which consists of an FFC that resists muscle 
contractions. These counteracting forces stiffen the body or limb. Hydraulic muscles provide the living organism's power source. There are several types of muscle arrangement combinations. Muscles form small rings around the coelomic cavity, as in the earthworm. Muscles radiate out from the center of the body cavity, and sea anemones have these muscles near the top of the head, where their arms radiate out (as in squid and cuttlefish). Muscles run longitudinally, as with the tube feet of starfish, and muscle fibers may be either crisscrossed or in a spiral configuration. In addition, certain exoskeleton arthropods, such as spiders, box mites, and whiptail scorpions, have hydraulic mechanisms in their legs. Although different from mollusks, the hydraulic structures are comparable in that the exoskeleton contains similar hydrostatic skeletal structures. Arthropods walk with their feet and the cavity contains the body fluid, which is under pressure in the muscle cavity and changes to transmit the hydraulic pressure.

Here, we summarize characteristics of biological hydraulics.

(1) Energy transfer and conversion

In the biological hydraulic drive, energy is transferred directly and varies significantly with the animal (Fig. 1), and the force generated by the muscle is transmitted directly through the working medium. Therefore, the biological hydraulic system is direct and efficient. Engineered hydraulic systems are often driven by multiple forms of energy conversion, resulting in considerable energy loss.

(2) Movement mode

The main components of engineered hydraulic systems are the hydraulic motor (rotary motion) and the hydraulic cylinder (linear motion). By contrast, the executing components of an organism rarely rotate and basically exhibit a reciprocating motion similar to that of hydraulic cylinders. In the process of execution, the large deformations of the body cavity and the vascular flexible body are coupled together, as in earthworms. During the driving process, the muscle force compresses the cavity or the side of the vessel, causing it to deform. The working medium in the cavity or in the blood vessel transfers the force to the other side to produce deformation and transmit force. In the biological hydraulic drive process, the generation and transmission of power are coupled together by the large deformation of the muscles, cavities, or blood vessels. This process is similar to the coupling effect of the hydraulic pump and the actuator in an engineered hydraulic system. This flexible drive transmission integration mechanism is called the muscle hydraulic cylinder system.

(3) Environmental friendliness

The working medium of an engineered hydraulic system is hydraulic oil, and transmission performance is closely related to this medium. Engineered systems have specific requirements for the oil, such as a specific viscosity and antioxidation ability. However, hydraulic oil can have negative environmental impacts. Leakage can result in the pollution of the environment, and this is a challenge for the successful use of hydraulic transmission technology. The use of hydraulic oil also creates a unique fluid noise that affects the environment. Organisms use body fluids or water in vitro as a working medium, which is environmentally friendly, cheap, and inexhaustible. Organisms are also typically lightweight, and their systems have a high power density and high reliability, factors that are prized in engineered hydraulic systems. Researchers have used filtered fresh water or sea water instead of mineral oil as the system working medium, which has resulted in energy conservation; water is also pollution-free and has the potential for further development.

(4) System pressure grade

The high pressure and speed of hydraulic components can significantly reduce the weight of 
the components and systems, improving the power density. The development of this capability is an important trend in the progress of hydraulic transmission systems. However, high pressure and speed can lead to a series of problems, such as a decrease in the system energy conversion efficiency, enhancement of fluid-solid coupling, and aggravation of the vibration noise. In contrast, biological hydraulic transmission systems tend to meet the animal's needs, reasonably control the pressure range, and lower the pressure on the organism's body. The pressure in the foot of a spider is on the order of $\mathrm{kPa}$. Even considering the size factor, the organism uses a low fluid pressure and speed to achieve efficient driving. Creating a system like that of the spider's would be of great value to engineers.

(5) Energy supplementation method

Engineered hydraulic systems must have a continuous power input and a cooling system for the working medium to maintain a stable system. Animals are powered by safe, biocompatible, and renewable fuels such as sugars and fats. Messner et al. presented a discussion of the energy requirements of biomimetic robots ${ }^{[26]}$. The limitations of fuel storage and usage and the need to improve the efficiency of motion in robots are critical issues. One of the major advantages that living organisms have over robots is their ability to harvest energy from the environment by eating. Moreover, an organism can continue to carry out everyday activities without refueling (eating) for several days. In comparison, most robots operate for less than one hour on their carried fuel; the cost of transport is 15 or 20 times greater than that for animals. In the case of the MIT Cheetah robot, the overall cost of transport is similar to animals of a similar scale, and its mechanical power is only $20 \%$ of the total power consumption. Seventy percent of the power consumption is dissipated through heat in the process of converting energy to mechanical work.

\subsection{Prototypical features of biological hydraulic systems}

The hydraulic system's power element is the hydraulic pump. Consider, for example, the positive displacement hydraulic pump, in which the pump is composed of several sealed working volumes. These sealed working volumes can achieve periodic oil absorption and oil pressure processes that constantly change. In the distribution mechanism, the oil suction chamber and pressure chamber are separate and have an appropriate seal that ensures that the hydraulic pump has a continuous suction and oil discharge. Inspired by those mechanisms, we deduced that the existence of a hydraulic mechanism in a biological drive requires three necessary features: a power source, cavity, and working medium. The power source is similar to the hydraulic pump, and the cavity is similar to the hydraulic cylinder, which is a necessary condition for producing deformation. The working medium is similar to the hydraulic fluid. This concept is similar to that found in fluiddriven artificial muscles, which contain a compressible skeleton, flexible skin, and fluid medium [27].

The power source of biological hydraulic transmission systems is always the muscle. Figure 3 shows a variety of animal muscle morphologies. The musculature is elaborately arranged so that both the diameter and length of the cylinder can be actively controlled. The common muscle fiber orientations found in FFCs include circular musculature, radial musculature, and transverse musculature. ${ }^{[28]}$. Moreover, the walls of many FFCs are reinforced with layers of connective tissue fibers that control and limit shape change ${ }^{[29]}$. The circular musculature and a pressurized cylinder are reinforced with two types of fibers, as demonstrated in Fig. 3A. Contraction of circular, radial or transverse muscle fibers will decrease the diameter, thereby increasing the pressure, and because no significant change in volume can occur, this decrease in diameter must result in an increase in 
length. Following elongation, shortening can be caused by the contraction of the longitudinal muscle fibers, re-expanding the diameter, and thus re-elongating the circular, radial, or transverse muscle fibers. The longitudinal muscle fibers and the circular, radial, or transverse muscle fibers thus function as antagonists in a manner that is analogous to muscles on either side of a joint in an arthropod or vertebrate.

The working medium can include blood, hemolymph, intracellular fluid, or seawater. Through the working medium, a hydraulic mechanism can be produced in an organism, as shown in Fig. 4. Figure 4A shows the internal and external circulation of the shell 3-dimensional velocity profile of seawater in living organisms ${ }^{[30]}$. Figure $4 \mathrm{~B}$ shows the change in the external pressure during the swimming of a jellyfish [31]. Figure $4 \mathrm{C}$ shows the flow development of the colored ink during the swimming process of Clione limacine. Figure 4D shows the flow of a spider's foot ${ }^{[32]}$. A working medium is incompressible and thus maintains a constant volume. A cavity is an important sign of the hydraulic mechanism. Figure 5 summarizes the hydraulic cavities found in several animals, including five major types: a cavity surrounded by muscles and blood vessels in the foot of the arthropod, called the lacuna; the digestive cavity of insect larvae, the intestine, which is a multifunctional organ; the mantle cavity, contained in Cephalopoda, Gastropoda, bivalves, and other mollusks; the coelom or hemocoel; and the gastrovascular cavity in marine animals. A range of relatively soft-bodied animals that utilize hydraulic mechanisms is shown in Table 1.

\section{Locomotion of natural hydraulic driving systems}

The biological hydraulic system is used in multiple movement methods, such as those involved in multilegged walking, jet propulsion, excavating, crawling, and adsorbing. These systems are quite different from those of many muscle-driven organisms and bionic robots [33] (which are quite different from the rotational movement of electromagnetic motors). In the next section, we discuss the various categories of animals to provide clarity about how biological functions are driven by their unique hydraulic systems.

\subsection{Arthropods}

The femur-patella joint and tibia-metatarsus joints, which are parts of slender $\mathrm{C}$ (bow)-shaped legs (Fig. 6A [34, 35] ), are purely hydraulic joints $[\underline{36}, \underline{37}]$. Hydraulic pressure is generated in the spider's body during circulation, and then the pressurized hemolymph flows in the lacunae ${ }^{[37]}$, as shown in Fig. 6B, whose cross sections are strongly influenced by intrinsic muscle activity. The joint membrane consists of special bellows-like tissue that is folded to prevent unfavorable opposing torques during joint extension, in contrast with isotropic membranes (black arrows in Fig. 6C ${ }^{[38]}$ ). The spider's circulatory system, which consists of the hemolymph vascular system in combination with a complex system of sinuses and lacunae, is composed of the central pumping organ, the heart, and arteries [39]. Figure 6E shows the details of the system from Huckstorfa's work [40]. Shultz proposed that the suspensor muscles of the endosternite generate most of the prosomal pressure in "hydraulic" arachnids ${ }^{[41}, \underline{42}$. Hence, the hemolymph pressure in the prosoma may be increased by both muscle sets and the extrinsic and intrinsic leg muscles, which possibly play a substantial role [42-44] (Fig. 6D), and by the musculi laterals (ml) or endosternal suspensor (es), pleura (pl), and abdominal sac (as). The mechanism of pressure in the opisthosoma is also complex. It is believed that the contraction of the abdominal sac that is caused by the tubular heart contributes to the decrease in volume and increase in pressure ${ }^{[44-47]}$.

In addition, up to four pairs of dorsoventrally running muscles can occur in the opisthosoma, 
which is probably also involved in hydraulic pressure production ${ }^{[48,49]}$. Furthermore, it seems clear that several other muscles are able to compress the opisthosoma and may contribute to increasing its fluid pressure ${ }^{[44}, \underline{50]}$. The pressure distribution in the spider's body and leg is necessary for circulation. The hemolymph pressure in the legs is slightly higher than that in the prosoma, and the pressure in the prosoma is slightly higher than that in the opisthosoma, but the differences are small [51-53]. The pressure is distinctly different between the resting and active states. Prosomal pressures can reach approximately $2-3$ times the resting values during walking and may reach peaks of almost 50 times the resting pressure during maximum activity. In the legs, the maximum values may be even higher. The flow distribution in both the prosoma and opisthosoma and in the leg may be controlled and regulated by valves ${ }^{[54]}$. Furthermore, the spider has highly sensitive organs located on its leg joints (black arrows) for the detection of external forces and vibrations generated by prey, mates, or predators $[55-58]$.

After investigating a large spider, Weihmann reported that although hydraulics are globally active during accelerated locomotion, as in starts and jumps, large spiders apparently do not use it primarily for propulsion ${ }^{[59]}$. This observation indicates that hydraulic pressure is also an important component of locomotion in many arachnids and may function in synergy with transarticular sclerites ${ }^{[60]}$. The whip scorpion is a typical arachnid that utilizes this mechanism. Sensenig et al. tested the extension torque contributed by the elastic recoil and hydraulic pressure in the chela (tibia-tarsus) and femur-patella joints of scorpions and the patella-tibia and tibia-tarsus joints of sun spiders and found that the transarticular sclerites function as spring-like extensors in the pedipalps of scorpions and sun spiders ${ }^{[61]}$. Mites, with the fastest relative speed and stride frequency documented among running animals ${ }^{[62]}$, produce holding forces on horizontal rough surfaces of up to 1180 times their weight; on vertical rough surfaces, they can pull 530 times their weight, effectively involving only two pairs of legs ${ }^{[63]}$, which might be involved in the hydraulic mechanism [64]. Wauthy reported the jumping capacity of a box moss mite. When the animal is propelled backward, its body simultaneously spins in a forward direction. It is thought that at take-off, the hind legs initiate a forward rolling of the body, which is powered by the internal hydraulic pressure, before the hook disengages from the shield rim and triggers a sudden backward impulse [65].

Spiders can be found in various environments, indicating that they have excellent environmental adaptability and intelligent driving ability. The jumping spider ${ }^{[66]}$ is able to jump a distance of more than 50 times its own length. Cupiennius salei ${ }^{[66-69]}$, although it tends to generally be inactive, is much faster than many other arachnids when provoked. Fish spiders, also called semiaquatic spiders ${ }^{[70]}$, are generally well-adapted to rapid movement to catch prey. Trap-jaw spiders hunt by sneaking up on their prey and rapidly snapping their mandibles shut, and a rubberband-like movement enables the whipping motion ${ }^{[71]}$. The prey of crab spiders may be several times larger than the weight of the predator ${ }^{[72]}$. The spider was chosen as an example to illustrate a mechanism that has yet to be thoroughly investigated. The hydraulic system in the spider is an intelligent closed-loop hydraulic system. The system is well organized and precisely controlled, and it has the ability to adjust quickly to allow movement. The system has a rapid switching mechanism between the real-time mode and ideal mode for efficient transmission and locomotion. By employing this mechanism, the system can realize the lowest energy consumption and produce the greatest functional output.

\subsection{Insect larvae}

Although the biomechanics of terrestrial soft-bodied animals are often discussed as a common 
group, the body plans of worms, mollusks, and caterpillars differ in important ways. In fact, while sometimes confused with worms, the larval stages of insects have a completely different anatomy and locomotion strategy. Burrowing species, such as fly larvae (maggots) and sedentary Hymenoptera larvae (e.g., wasps), generally lack limbs, but butterfly and moth larvae are highly active climbing animals with well-developed gripping appendages called prolegs.

The internal organs of dragonfly larvae include highly effective hydraulic mechanism [구 . Larval digestion, respiration, prey capture, and locomotion all employ liquid pressure changes both within and outside the body. By closing a sphincter at the entrance to the intestines and contracting the walls of the abdomen against the water-filled rectal chamber, the resulting hydraulic pressure thrusts the feeding "labium" toward its prey. This action raises the internal body cavity pressure and pushes the labium out in a strike that takes 10 to 30 milliseconds. The amount of internal pressure generated is approximately $6 \mathrm{kPa}$. Considering that large nymphs weigh only $100 \mathrm{mg}$, the idea of generating almost 1 pound of pressure with their rear is impressive. An additional amazing function is locomotion by jet propulsion. By reversing the process (closing the anterior (or "head end") sphincter and contracting the abdomen), water can be fired out of the anus at up to $50 \mathrm{~cm} / \mathrm{s}$, propelling the animal forward. The preying activity and rapid movement are important for the organisms' survival; hence, a multifunctional system harmoniously accomplishing those two tasks could provide inspiration for the design of a robotic drive system.

Although the caterpillar body appears to be segmented, there are no internal divisions between these segments; rather, there is a single, continuous fluid cavity, called the hemocoel, surrounded by muscles ${ }^{[74]}$ (Fig. 7A). Simon et al. first described the movements of the internal tissues in Manduca using synchrotron-sourced X-rays. As shown in Fig. 7B ${ }^{[75]}$, the Malpighian tubules (MT) and gut-associated tracheae (GAT) can be seen moving forward in synchrony with the swing phase of the terminal prolegs. The movement of the gut contributes to locomotion by shifting the center of mass forward and by acting as a "visceral piston," which is helpful for extending the anterior segments and thorax forward. Presumably, the movements of the gut also result in a flow of hemolymph that contributes to local differences in pressure throughout the body cavity. The maximum forward crawling speeds of caterpillars are limited by the hydraulic design of the body and the peristaltic mode of operation of the segmental muscles. High-speed locomotive maneuvers can be achieved by reversing the direction of the normal peristaltic wave (from posterior-anterior to anterior-posterior), although the penalty is a dramatically reduced duty factor of the legs and potential instability [76]. The mechanisms by which larval insects control their movements are expected to reveal useful strategies for designing soft biomimetic robots.

\subsection{Mollusca}

The mollusks that employ hydraulic transmission include both marine animals, such as squid, and terrestrial animals, such as snails. However, there are clear differences in their driving mechanisms. The hydraulic mechanism of marine animals is primarily based on jet propulsion, and its working medium is often the surrounding water. In contrast, the working medium of terrestrial animals is their body fluid. Their hydraulic mechanism is manifested in the use of the pressure changes within the body to drive movement by an enlarged pedal sinus $[\underline{77}, \underline{78]}$. In addition, mollusks use hydraulics for excavating [79, $\underline{80]}$.

Some species of Mollusca employ hydraulic functions, although they do not seem to be involved in locomotion [81]. There is a report that the predatory pteropod mollusk Clione limacina 
catches its prey by using specialized oral appendages called buccal cones, and its eversion and elongation are carried out by internal fluid pressure; therefore, this is a hydraulic phenomenon [82]. The evolution of venomous animals has been a subject of considerable interest to biologists. As described in the literature ${ }^{[83]}$, high-speed hydraulic prey capture represents a key adaptation in the evolutionary and ecological radiation of Conoidea. The mechanism is a versatile method for the injection of toxins, allowing these snails to expand the range of prey types exploited.

\subsubsection{Cephalopods}

When cephalopods attack or escape, they employ very large body and shape deformations, making the related flow-structure interaction problem radically different from that of rapidly maneuvering rigid-hull vehicles. Marine animals can rapidly achieve a high acceleration, which is impressive, particularly because it is achieved within a heavy liquid [4]. The squid employs a hydrostatic skeleton with an extensive fluid-filled cavity to achieve jet propulsion. There are circumferentially arranged muscles in the walls of the squid mantle (Fig. 8A ${ }^{[85]}$ and Fig. 8B ${ }^{[86]}$ ). As shown in Fig. 8C, water in the mantle cavity is pressurized by the contraction of these muscles and expelled as a jet ${ }^{[87]}$, and a change in the mantle's diameter occurs during jetting. After jetting, the squid refills its mantle cavity with seawater to prepare for the next jet event.

Squid are the largest jet propellers in nature as adults, but as paralarvae, they are some of the smallest, faced with the inherent inefficiency of jet propulsion at a low Reynolds number (Re). Squid paralarvae typically have rounded bodies, small fins, and relatively large funnel apertures, whereas juveniles and adults operate at a higher Re and generally have more streamlined bodies, larger fins, and relatively small funnel apertures. These morphological changes and varying flow conditions affect the swimming performance of squids. Ecological pressure to maintain maximum velocity may lead them to increase the aperture size, which reduces efficiency $\left[\frac{88-90]}{\text {. Amphioctopus }}\right.$ marginatus, the notorious coconut octopus, is known for its unusual behaviors, including bipedal walking and tool use. This species is the first example of bipedal locomotion using a hydrostatic skeleton rather than rigid support ${ }^{[91]}$.

\subsubsection{Burrowing mollusks}

The fluid-filled cavities present in the foot of many burrowing mollusks serve an important function in movement during burrowing in bivalves [22-95], scaphopods, and gastropods. The hydraulic mechanism allows the force of muscle contraction to be transmitted over large distances and allows major shape deformations.

The digging activity of Ensis arcuatus shows six stages, together termed the "digging cycle," which are repeated cyclically and are similar to those of other burrowing bivalves. When bivalves burrow, the dilated foot serves as an anchor while the shell is pulled down into the sediment, and the shell functions as an anchor during probing and extension of the foot (Fig. 9, burrowing in mud, A, and sand, B). The shell, the basis of a fluid/muscle system, consists of two separate fluid-filled chambers: the hemocoel and mantle cavity. In the hemocoel, this pressure gives rise to the characteristic bulbous form of the foot, which ensures a secure anchorage so that at pedal retraction, the shell is drawn down. In the mantle cavity, the pressure produces powerful jets of water, which assist in the movement of the shell by loosening the adjacent sand. Stage iii shows that the shell closes and drives additional internal fluid (blue arrow) into the foot and drives fluid out from the mantle cavity (cyan arrow). This dual-anchor system also describes the behavior of many softbodied burrowers [96]. With a hydraulic mechanism, however, dilation of the tip occurs by the 
displacement of fluid, and once anchorage is established, shortening occurs.

\subsection{Worm-like animals}

Worm-like animals, primarily Annelida and Nematoda, depend on hydrostatic skeletons with FFCs to support their bodies ${ }^{[97-100]}$. These skeletons usually comprise both circular and longitudinal muscle fibers, and the organisms' body shapes are typically cylindrical.

The earthworm has a clearly segmented structure and noticeable fluid-filled compartments. Each segment has its own discrete fluid-filled coelomic compartment. The parts of the earthworm, Lumbricus rubellus, are shown in Fig. 10A ${ }^{[101]}$ and include the following: a) the gut section that follows the thickened glandular clitellum, called the posterior alimentary canal (PAC); b) a crosssection of the posterior section of the earthworm; c) a detailed cross-section of an earthworm with the fluid-filled coelom; and d) a diagram illustrating the morphometric measurements made on Lumbricus terrestris $[102,103]$. As discussed above, the earthworm's body function as a peristaltic pump (Fig. 10B). In contrast to animals with rigid skeletons, in this mode of locomotion, there are no changes in the joints' angles between the rigid links; instead, there are changes in the dimensions of the body regions. A region may shorten while increasing in diameter or elongate and contract radially depending on the hydrostatic skeleton.

Annelids have a segmented body in which each segment is separated by septa. Their FFC is a true coelom; that is, the cavity is completely lined with mesoderm-derived tissue ${ }^{[104]}$. Although the earthworm and the leech are both annelids, their FFCs have several differences. The earthworm has a clearly segmented structure and noticeable fluid-filled compartments, as shown in Fig. 11A. Waves of alternating activation of the longitudinal and circular muscles produce a peristaltic wave, moving from tail to head, which is used for locomotion and burrowing. The leech, which belongs to the Hirudinea, has a dramatically reduced coelom and lacks septa, as shown in Fig. 11B. In nematodes (the round worms, such as Ascaris), there are no circular muscles; instead, there is a fibrous cuticle that prevents the shortening of the animal. The whole interior of the animal, inside the fibrous cuticle, acts as a hydrostatic skeleton. These animals have an FFC in the form of a pseudocoelom (i.e., the body cavity is not completely lined with mesoderm), in contrast to the annelids. Their muscle composition allows undulatory motion but not peristalsis due to the lack of circular muscles.

\subsection{Cnidaria}

Jellyfish are among the most awesome marine animals, and they perform a spectacular "dance" in water. Polyps, such as sea anemones, sea whips, corals, and hydroids, are classic examples of animals that rely on a hydrostatic skeleton. Fig. 5E [105, 106] clearly shows the fluid-filled cavity, called the gastrovascular cavity or coelenteron, which also acts as the gullet, stomach, and intestine. The body of a sea anemone is in the form of a hollow column that is closed at the base and equipped at the top with an oral disk that includes a ring of tentacles surrounding the mouth and pharynx. When the mouth is closed, the water in the internal cavity - the coelenteron-cannot escape, and thus, the internal volume remains essentially constant.

The locomotion of jellyfish is very complex, although the motion of jellyfish is very simple. Generally, the flow of jellyfish created during each propulsive cycle consists of a toroidal starting vortex formed during the power swimming stroke and is followed by a stopping vortex of an opposite rotational sense generated during the recovery stroke. These two vortices merge in a laterally oriented vortex superstructure that induces flow both toward the subumbrellar feeding 
surfaces and downstream. Figure $12^{[107]}$ shows the key concepts of fluid transport in the jellyfish and their in vitro implementation. In Fig. 12A, panels a-d show the jellyfish stroke cycle, controlled configurational change, stroke kinematics, and fluid dynamics. Figure 12B ${ }^{[108]}$ shows an analysis of the pressure dynamics during the oblate jellyfish rowing locomotion. The horizontal scale lines indicate $1 \mathrm{~cm}$. The mechanism of locomotion, especially the flow pattern, is still a popular research topic for many scientists and is undergoing active investigation $[109-111]$. The unique feature of marine organisms such as jellyfish is not their high speed but their high efficiency. It has been reported that jellyfish possess the most highly efficient locomotion of all animals studied.

\subsection{Echinoderms}

Echinoderms share a fundamental characteristic: the presence of a WVS with an external opening. This system, in particular, remains a unique application of a hydraulic system, generating pressure gradients that are able to command hundreds of tiny tube feet to crawl over uneven surfaces and feed on tough-shelled prey, such as mussels. Figure 13A shows the details of a WVS in starfish; the WVS of other echinoderms is basically similar to that of the starfish. The system generally comprises the madreporite, stone canal, ring canal, radial canal, lateral canals, and tube feet. The starfish's WVS provides the water pressure that operates the animal's tube feet. From the madreporite, water moves into the ring canal, then into the rays through the radial canals, and finally to the tube feet. The canals are similar to a network of water pipes attached to the tube feet. Water also exits the body through the madreporite. The tube feet are arranged in two lines along each arm and are connected to the WVS. When water starts moving, the tube feet sometimes wave randomly, after which coordinated movements are organized. Thus, the movements of the tube feet are not under strong control by a "central nervous unit," but occur through the interplay of external stimuli, resulting from the activity of each tube foot and the internal state, such as biases toward movement in a certain direction caused by the arms' activities.

The tube feet are of particular interest. Starfish tube feet are subdivided into the knob-ending (Fig. 13B a: the SEM view of the external morphology), simple disk-ending (Fig. 13B b), and reinforced disk-ending tube feet (Fig. 13B c) [112, 113]. Figures 13B d-f show the histological organization of the three tube foot morphotypes, which possess a similar histological organization $[114,115]$. The tube feet consist of four tissue layers that include the following, from inside to outside: a myomesothelium surrounding the water-vascular lumen, connective tissue layer, nerve plexus, and epidermis covered by a thin cuticle ${ }^{[116]}$. Simple disk-ending tube feet are primarily used by most valvatid species for locomotion. Reinforced disk-ending tube feet are used in locomotion, for attachment to the substratum, and, in some species, as an opening for the bivalve that allows for feeding. Several features of the tube feet are shown in Fig. 13C (which exhibits the tube feet of the sea urchin Paracentrotus lividus [117]). Figure 13C a shows an enlarged view of the tube feet; Fig. $13 \mathrm{C} \mathrm{b}$ shows the SEM view of the external morphology; and Fig. 13C c shows the histological structure of the tube feet.

In addition, the WVS in echinoderms, such as starfish, employs hundreds or thousands of tube feet to achieve locomotion. During locomotion, the tube feet undergo repeated stereotyped stepping cycles. An individual tube foot first elongates and bends at the base so that the tip of the foot points in the direction of motion. Once the tube foot is fully protracted, it bends downward until the tip contacts and attaches to the substratum. The tube foot then bends at its base and moves the animal over the point of attachment. In the final phase of the cycle, the tube foot releases its attachment and retracts. The cycle is then repeated. The connective tissue fibers observed in both the tube foot and 
the ampulla wall play an important role in the mechanics of the tube foot and the ampulla. Consider first the requirement that an increase in pressure (due to contraction of the ampulla) causes the elongation of a tube foot. The crossed-fiber helical array of connective tissue fibers described here reinforces the wall so that an increase in pressure causes an increase in the length, rather than the diameter. The valves located at the opening of the lateral canals into the tube feet also have an important function in the WVS of asteroids. The valve flaps are arranged so that an increase in pressure in the tube foot-ampulla complex extends to close the valve, preventing the loss of fluid from the tube foot.

\subsection{Chordata}

Salps have a complex lifecycle, with an obligatory alternation of generations. The solitary life history phase, also known as an oozoid, is a single, barrel-shaped animal that reproduces asexually by producing a chain of tens to hundreds of individuals, which are released from the parent when they are small, as shown in Fig. 14A. The chain of salps is the "aggregate" portion of the lifecycle. The aggregate individuals are also known as blastozooids, as shown in Fig. 14B. Salp jet propulsion is one of the most efficient types in the animal kingdom. Salps are unique in possessing incurrent and excurrent siphons on opposite ends of the body, allowing unidirectional flow and reverse swimming during escape. In comparison to squids, salps have siphons on the opposite ends of the body; thus, losses due to intake are small. Salps swim continuously using a high-volume, lowvelocity jet, which also drives a feeding current. In contrast, squid do not need to swim continuously and can also rely on fin motion to aid in swimming. For squids, speed and maneuverability may be enhanced at the cost of propulsive efficiency. Furthermore, the bodies of salps are generally aggregated and are arranged parallel to each other so that the plurality of the jetting fluid between salps has coupling effects that are conducive to propulsion.

Figure 14C and Fig. 14D illustrate the structures and fluid flow through siphons in a solitary Pegea confoederata and an aggregate Weelia cylindrica, respectively. The arrows indicate inflow through the oral siphon (black) and outflow through the atrial siphon (gray). The propulsive jet for locomotion is created by the rhythmic compression of the muscle bands encircling the barrel-shaped body. Fluid enters the anterior oral siphon to fill the mostly hollow body of the salp. Next, the oral lips close and the circular muscle bands contract, decreasing the volume of the jet chamber so that fluid is accelerated out of the posterior atrial siphon. By the end of the jet period, the atrial siphon is closed, and the oral lips open to repeat the unsteady rhythmic process. Fast-swimming salps are characterized by an enhanced musculature, higher pulse frequencies, and linear, streamlined chain structures compared with those of slower, surface-oriented salps ${ }^{[118-120]}$. Figure $14 \mathrm{E}$ and Fig. 14F show the two in situ jet wake structures made visible with fluorescein dye.

Comparative swimming performance among salps is of particular interest because swimming and feeding are achieved by the same pumping process. In the process of drawing fluid through the body of the salp during jet propulsion, fluid and associated particles pass through a mucous filterfeeding net. Particles between $1 \mu \mathrm{m}$ and $1 \mathrm{~mm}$, primarily phytoplankton, are captured by the net and conveyed to the gut. Therefore, a trade-off is established between these two fundamental activities.

\section{Bio-inspired pressurized liquids in robot actuation}

Future generations of robots will be bio-inspired and will have soft bodies composed of soft materials, actuators, and sensors, which could expand the requirements for adaptation and safety in the realm of human-robot interactions ${ }^{[121]}$. Wahner et al. developed the first full-bodied robot-driven 
by a pneumatic mechanism [122]. The actuator types of soft robots can be divided into three types: variable pressure actuation based on fluid, actuation based on the deformation of intelligent materials, and underactuation based on variable cable length [123]. Actuation based on the deformation of intelligent materials generally includes an ion-exchange polymer metal composite (IPMC), dielectric elastomeric actuator (DEA), shape memory alloy (SMA), or responsive hydrogel. A variable pressure actuator based on a fluid has several advantages over these materials in terms of the output force, power density, and ease of control [124-127].

In nature, organisms actuating by pressurized liquids have soft bodies and excellent flexibility, and these creatures can provide a knowledge base for the development of fluidic actuators. Fluidic actuators can be divided into hydraulic and pneumatic actuators based on the selected medium, which primarily includes pneumatic artificial muscle (PAM) [121, 128, 129], fluidic elastomer actuators (FEAs), and other hydromuscles $\stackrel{[130]}{ }$. Generally, liquid serves as a sufficient soft robot actuator with the advantages of incompressibility, high-response frequency, and minimal leakage. However, its gravity effect will affect the modeling and control of the soft robot. Fiber-reinforced actuators achieve a specific type of motion by selecting the appropriate placement of reinforcements, and it is possible to achieve multiple motions, including bending, extension, and twisting [131-133]. The motion of a PneuNets actuator is controlled by modifying the embedded chambers and material properties of their walls. PneuNets bending actuators can achieve only a bending motion. Examples of pneumatic actuators that utilize PAM, PneuNets, and liquid actuators using FEA are shown in Fig. 15 and Fig. 16.

Compared with traditional robots, hydraulic transmission devices, especially fluid drivers, have important applications and outstanding development prospects in bionic walking/jumping robots and dexterous manipulators/arms. Fluidic actuators combined with artificial intelligence and the application of portable and wearable machinery also provide new avenues for modern hydraulic transmission technology to serve as intelligent equipment.

Here, we briefly summarize the current status and development trends of fluidic actuators while taking into consideration natural pressurized liquid actuation.

(1) Promotion of transmission capacity

Driving-transmission technology is related to the transformation and power transfer of a robot's energy, which is an indispensable technology to promote innovation in robots. PAMs are limited by their traditional cylindrical form factors, which must increase radially to improve contraction force generation ${ }^{[134]}$. Hence, soft fiber-reinforced actuators have been widely investigated and developed, and include PAM fascicles for their high force and reliability [135], fiber angles of the deformation of fiber-reinforced soft fluidic actuators [136], and EBPAMs [136, 137]. It is important to consider a fiber-reinforced [138], internal flow structure, fluidic structures with fiberreinforced elastomeric enclosures [139], and fabrication from elastomer films with embedded fluidic channels ${ }^{[140]}$ for fluid actuators ${ }^{[141]}$. In the future, it will be important to study the fluid state, load variation, and structural optimization of fluidic actuators combined with computational fluid dynamics (CFD), fluid-solid interactions, and nonlinear multidisciplinary optimization.

(2) Research on the matrix material of fluidic actuators

Fluidic actuators, especially the hydraulic type, are actuated by the deformation of elastic matrix materials. Therefore, the performance of fluid actuators is also dependent on the preparation and structure of high-performance soft materials. Inspired by the penis as a variable-volume hydrostatic skeleton ${ }^{[142]}$, Loepfe et al. created a new type of mammalian vein-inspired soft silicone 
pump [143]. Soft silicone is a key factor that determines the performance of a pump. Roche et al. developed a pneumatic artificial muscle arranged at a certain rotation angle and manufactured an artificial heart that can produce similar cardiac contractions [144]. A great advantage is that the structure of this actuating integration breaks through the limitations of artificial muscle action, lending itself to achieve bending, twisting, extension, and flexion with nonrigid materials. The flexible mechanism is similar to the muscle in a biological hydraulic transmission, namely, the power source. In fact, musculature has been deemed the "prototypical soft actuator" because it can achieve fast, strong actuation and remarkably complex patterns of movement. Therefore, it is necessary to develop soft materials with a high energy density, large deformable actuation, and strong compliance. In the future, the development of soft intelligent material ${ }^{[145,146]}$ and biomimetic material $[147,148]$ will be a priority. Furthermore, matrix material can be developed into hydraulically amplified self-heading electrostatic (HASEL) actuators $[\underline{149}, 150]$, which combine intelligent materials, responsive hydrogels, fluidic actuation, PEA, and DE. This progress will lead to novel development trends for robotic actuators.

(3) Intelligent structures

Research into soft robots has also generated new requirements for flexible electronic technology and has opened up a broad range of possible applications. The closed-loop control of fluid actuators is still very challenging due to the lack of robust, reliable, and inexpensive sensors that can be integrated onto highly deformable actuator structures [151]. Several efforts are being directed toward these goals to impart sensation for feedback control via flexible or stretchable sensors that can be integrated with the soft bodies of robots without hindering their motion or reducing their safety ${ }^{[152,153]}$, such as the Smart Braid McKibben Muscles ${ }^{[154]}$, which are a PMA embedded with a resistive flex sensor ${ }^{[155]}$. To achieve more effective control, combining the 3D/4D printing ${ }^{[156-158]}$ of structures directly to achieve the integration of sensors and fluidic actuators is a promising approach and has become a research hotspot $[159,160]$. To create efficient systems like those found in animals, researchers should combine or fuse the drive element, sensor element, and control system into a flexible mechanism that perceives changes in its external environment and internal state. The information from the engineered system's environment can be identified and inferred through its own mechanism to determine a reasonable decision for actuating the device and its response. This concept of an engineered system that functions like an organic system has become an important trend in the development of fluidic actuators.

The process of developing a high transmission capacity and intelligent fluidic actuators consists of three steps: analyzing biological hydraulic transmission functions, revealing the mechanism of energy transformation, and reproducing the fluid transmission capacity of organisms. The composition of the muscle system, dynamic changes in the transmission process, transmission of energy, and control of the fluid medium in the movement process are significant research areas. The development of fluidic actuators is of great value for the design of an effective robotic structure. As shown in Fig. 17A, a soft artificial muscle was implemented as a bicep to lift a skeleton's arm to a $90^{\circ}$ position at the elbow ${ }^{[161]}$. Figure 17B shows a bionic soft artificial heart that can imitate the twisting motion of the human heart ${ }^{[144]}$. In Figure $17 \mathrm{C}$, the actuator acts as the arm to lift a volleyball of a standard size and weight $\frac{[162]}{}$. Another key component of the bionic actuator is the manipulator, as shown in Fig. 17D [163]; the manipulator, which groups micro-electrohydraulic actuators to drive the five fingers of the robot hand, integrates a design weight of only $2 \mathrm{~kg}$ and an output force up to $260 \mathrm{~N}$, giving full play to the advantages of the high power density of a hydraulic 
drive. Future research will lead to reductions in the structure of artificial muscle and more powerful and intelligent systems that will achieve functions similar to biological muscles. At that time, the structure of a robot will not be limited by existing actuators, but will become more flexible, powerful, and truly integrated with human computers.

\section{Development trends of the hydraulic transmission system and biomimetic}

\section{studies}

Technological inventions involve new technologies, structures, and processes developed in the course of simulating the functional characteristics and principles of biological examples. These innovations form the foundation of bio-hydraulic bionics and are the key to engineering innovation. The discovery of natural systems involves the revelation and explication of the mysteries of the natural environment, ecology, and phenomena, which are also the primary goals of bionics. Natural systems are the ultimate source of technological innovation and engineered creations. Many valuable functions have been discovered in biological hydraulic transmission systems, such as the intelligent spider drive system, hybrid locomotion in the box mite, elaborate multifunctional systems in dragonfly larvae, fast-moving and transient acceleration by jet propulsion, the soft overcoming the hard during burrowing, highly efficient systems that operate at low speeds (like those found in jellyfish), and the combined drive-by units of siphonophores. Technological innovation promotes further discoveries and helps to reveal the intrinsic mechanisms and laws of energy conversion by biological hydraulic transmission, which in turn drives the discovery of new concepts, ideas, and the creation of bionic designs. Engineered creations are the ultimate goal of bio-hydraulic simulations. The engineering process also represents an approach to addressing the challenges facing existing hydraulic systems. We anticipate the incorporation of more bio-hydraulic systems into future hydraulic technologies, which may involve a combination of materials, structures, drives, controls, and other components. The biomimetic "map" presented by Vincent ${ }^{[164]}$ demonstrates the transfer from biology to engineering. Systems with natural origins can be scaled up into more powerful, engineered systems." $[165]$.

A motor drive mode was developed for use with an artificial intelligence. However, intelligent hydraulic or hydraulic transmission technology is still in its infancy, so intelligence is an important trend in the development of hydraulic systems. There are possible applications for hydraulic transmission technology in small- and medium-sized power (or even micro) equipment. Therefore, the advantages of a lightweight hydraulic transmission device promote the application of soft robots, and portable and wearable machinery. Hydraulic transmission technology can adapt to a complex environment and can be expanded to address the challenges of creating a clean environment. In addition, energy saving and consumption reduction are critical to human society and the development of industrialization. By addressing the weakness of a low energy efficiency, hydraulic transmission technology will become a long-term development demand.

The hot topics of hydraulic systems research include the following: intelligent and reliable hydraulic components and seals; lightweight and intelligent transmission systems; key technologies for driving digitalization; drive integration (valve, cylinder, feedback, and drive control), such as the compact hydraulic power unit (CHPU) used in exoskeletons ${ }^{[166]}$; and the application of additive manufacturing for hydraulic components, such as MIT's printable hydraulics [167]. The current development of hydraulic systems (Fig. 18) can provide a preliminary outlook for the role of biohydraulic transmission systems. 
(1) Environmentally friendly transmission systems

There are three main aspects of the environmental unfriendliness of engineered hydraulic systems.

a. High power loss and increased energy consumption

Energy consumption is inevitable when the transmission medium flows in pipes and components; it includes not only the losses due to the frictional resistance, one-way resistance, and local resistance, but also the power loss from internal leakage. The power mismatch in the design of an oil-hydraulic circuit itself also results in a large power loss and low efficiency.

b. Greater noise and vibration

The volumetric pump used in a hydraulic system applies a closed-volume alternation change to achieve oil absorption and oil drainage. Hence, the output flow of a hydraulic pump pulsates, which causes pressure pulsation and noise in the hydraulic system.

c. Increased environmental pollution of the working medium

The majority of hydraulic systems employ mineral-type and nonflammable hydraulic oil as the working medium; however, oil leaks, and discarded oil can cause serious ecological pollution due to its extremely low degradation rates.

As noted above, in comparison with bio-hydraulic systems, engineered hydraulic systems usually utilize high-pressure and high-speed fluids to increase their power density. Figure 18 shows the pressure increases in hydraulic control systems. The pressure gradually increases above the baseline pressure of $35 \mathrm{MPa}$ to higher than $50 \mathrm{MPa}$. When the flow speed of the working medium is greater than $15 \mathrm{~m} / \mathrm{s}$, it presents a series of challenges to the system's pressure compensation ability, system cooling, seals, reliability, vibration, and noise control.

There are three environmentally friendly aspects of bio-hydraulic systems that may effectively avoid the above problems.

a. High driving efficiency

Because of the survival of the fittest in natural selection, organisms must obtain the maximum energy efficiency and consume the minimal amount of energy for survival. Organisms, such as spiders and squid, utilize fluids inside or outside of the body to carry out various actions, generally applying a low pressure to produce a high power output. In addition, some marine animals, such as jellyfish and starfish, exhibit a low speed but extremely efficient drive mode.

\section{b. Hybrid locomotion mechanisms}

The structures, materials, sealing mechanisms, vibration, noise suppression mechanisms, driving modes, and control mechanisms of bio-hydraulic systems are worthy of further study for different engineering needs. For example, box mites exhibit exceptional movement abilities, producing forces as high as 1180 times their body weight, and they are capable of turning with extremely high angular velocities (at relative speeds of $192.4 \pm 2.1 \mathrm{BL} \mathrm{s}^{-1}$, with stride frequencies as high as $135 \mathrm{~Hz}$ ). The cooperation between muscles and the hydraulic system clearly plays a decisive role in their ability to move well. Hybrid locomotion mechanisms can inspire the creation of a fast-moving, powerful robot.

c. No working medium pollution

The working medium adopted by bio-hydraulic systems is the same as that in its living environment. Therefore, the system will not pollute the environment during the discharge of the working medium, but will return the medium to its original environment. For example, squid and fish live in the ocean, so their discharge processes will not produce toxic or harmful substances or 
pollute the environment.

(2) Integrated power generation and transmission systems

Mechatronics technology can overcome the shortcomings of hydraulic transmission systems to further develop advances in hydraulic controls and to maintain competition with other forms of transmission. Mechatronics involves the combination of hydraulics and mechanical, electrical, electronic, computer, and network technologies to continuously pursue the development of drive integration. This integration technology can result in engineered systems with a small size, light weight, low energy consumption, high reliability, and intelligence by integrating servo-valves, hydraulic cylinders, hydraulic accessories, sensing systems, control systems, and other components together through new manufacturing methods. The progression of hydraulic transmission technology and the advancement of manufacturing technology are inseparable. Hydraulic components usually have complex internal cavities for oil circulation. For traditional manufacturing methods, the more complex the cavity structure is, the more difficult it is to manufacture, and the longer the production cycle. Current 3D printing technology provides a very good solution for such problems and is expected to have a great impact on traditional hydraulic component manufacturing technology and production modes. Integration can increase system power densities by making the component structures more compact and can enhance transmission efficiency by reducing the energy loss caused by pipeline mutations in the transmission process. The integration of manufacturing and hydraulic transmission technologies can also result in high integration-level components, reduce the risk of pipeline leakage, and improve the reliability of the system. Currently, the typical integrated smart actuators manufactured by the MOOG company have been applied to Bigdog, Atlas, HyQ, and other systems. However, there is a noticeable lack of consideration of the complex environment of the drive process. There are still many bottlenecks in the current key technologies of integrated hydraulic actuators, such as the fusion mechanism of the high-efficiency power transmission capability in multi-components; the detection of sealing, sensing, and state; and the control of intelligent compliance.

There have been many bio-hydraulic actuators that mimic the function of spiders, such as the "Smart Stick" [168-170], the flexible fluidic actuators developed by Schulz ${ }^{[1711}$, and the flexible fluidic actuators created by Landkammer et al. ${ }^{[172]}$. A pneumatic and tendon-driven spider robot was also developed $\left[\frac{[173]}{}\right.$. The bio-inspired fluidic actuator offers a high power/weight ratio. However, the output torque needed to advance the development of control systems is not easily achieved. The actuator's power density and output power are far less than those found in a living organism, and the same level of intelligence as found in a living creature have not yet been achieved.

Intelligent and integrated actuators are the key components of high-end equipment, and a biohydraulic drive can improve their development. The fluid-filled cavity skeleton is a highly integrated power generation and transmission system, and the body works as a pump and soft actuated-hydraulic cylinder by producing deformations. The relationship between the diameter and length in a fixed-volume cylinder is not linear, as demonstrated in Fig. 19A ${ }^{[174]}$, and a unit decrease in diameter when the cylinder is short and wide (from A to B) results in a small increase in length of approximately 0.6 units. A unit length change applied to a longer and thinner cylinder (from $\mathrm{C}$ to D) increases the length by more than 10 units. The subfigure (in the red box) shows the sequential jet propulsion procedure of a squid from (a)-(d); the body diameter will clearly decrease [175].

A biological system avoids the problems of an engineered integrated drive system through its own structure, sensing, and control mechanisms. Changes in the rules of the fluid cavity in the drive 
process of organisms provide many important references for the development of efficient topological structures in the flow field of integrated hydraulic actuators. Determining how organisms avoid sudden changes in the flow channel and reduce flow resistance to achieve lower energy losses would be a valuable development in engineering hydraulics. The biological seal is a highly desirable technology because seal failure can pose a serious threat to life; therefore, biological seals can also inspire the development of integrated hydraulic actuators. Furthermore, the intelligent control mechanisms associated with biological integration with the external environment are important and ensure that organisms achieve a high energy output with a low energy consumption and react to external stimuli. The efficient energy transfer and fusion mechanisms of biology, which can support the in-depth learning of biological functions, will be a continuing source of novel ideas.

(3) Digital hydraulic systems

Digital hydraulics have been widely regarded as the direction of future hydraulic systems, and their goal is to realize the precise control of actuators. In digital hydraulic systems, the motion characteristics of the hydraulic actuators (cylinder and motor) correspond to electric pulse performance, which means that the frequency of the electric pulse corresponds to the movement speed of the cylinder (or the angular speed of the motor), and the number of electric pulses corresponds to the movement stroke of the cylinder (or the motion angle of the motor). The accuracy of the actuators is almost unaffected by the effects of the load, oil pressure, or leakage. Digital hydraulic systems are a combination of intelligent hardware and big data, which form the core technologies of Industry 4.0.

The central philosophy of digital hydraulics is to convert a "continuous simulation-continuous fluid" into a "digital quantity-digital discrete fluid." During hydraulic transmission, the fluid is continuously changing; digital hydraulics establishes the mapping law of the movement of the continuous fluid and digital discrete fluid. This process reduces the problems of low efficiency, poor adaptability, and weak autonomy of traditional hydraulic systems. With the emergence of digital hydraulic systems, hydraulic technology has accomplished a qualitative leap, and the barriers to design, application, and maintenance have been greatly lowered. Digital hydraulic systems have already achieved great advantages in heavy-duty drive fields due to their increased power density.

Organisms can precisely control their own movements, whether they move at a high speed or low speed, and whether they are multilegged, or creep, swim, or dig. Precise control of motion requires complete mastery of the fluid transmission process. Hence, it is worth studying whether the detection and control mechanisms of the fluid state in the process of bio-hydraulic transmission are consistent with digital hydraulics and what we can learn from these processes. There are difficult problems that need to be urgently resolved, such as how to effectively obtain and control a highfrequency discrete digital fluid and avoid the pulsations and shocks caused by fluid motion; how to obtain real-time flow information through in situ measurements of the flow field; and how to make use of the morphological characteristics of the fluid in the transmission process to form a large database that can be used to understand flow patterns. Successful comparative analyses of the above problems with the mechanisms of bio-hydraulic transmission will lead to many fascinating results.

\section{Discussion}

The main challenges for advancing hydraulic transmission systems include the following: increasing energy efficiency, improving reliability, building smart components and systems, reducing size and weight, reducing environmental impact, and improving and applying energy 
storage and redeployment capabilities. While humans are tirelessly exploring and upgrading the hydraulic drive systems of engineering equipment, nature could inspire solutions to these problems.

Many organisms use muscle power to produce a pressurized liquid that underlies the mechanisms of hydraulic transmission. The existence of a hydraulic mechanism in a biological drive requires three necessary features: a power source, cavity, and working medium. The power source is similar to a hydraulic pump; the cavity is similar to a hydraulic cylinder, which is a necessary component for producing deformation; and the working medium is similar to a hydraulic fluid. Under these three conditions, a biological flow occurs inside the body or outside the body to meet the needs of the biological drive. Natural fluid power is always linked to hydrostatic skeletons. Hydrostatic skeletons do not possess rigid elements but rely on pressure to create stiffness. As with the exoskeletons of arthropods, the muscle, blood vessels, nerves, and lacuna produce hydraulic transmission mechanisms.

Biological hydraulic transmission systems often differ from engineered hydraulic transmission systems in many ways, such as in the energy transfer and transformation, movement mode, environmental friendliness, system pressure levels, and energy supplement mode. In engineered oilhydraulic systems, the pressure is generated by a high-speed rotary motion. However, in animals, it is generated by the beating heart or muscle contractions. In addition, animal body sizes vary from the very large to the very small. The highly efficient pumps and actuators used in engineering do not deliver adequate performance at centimeter scales and below. Hence, the locomotion of animals is attractive. In most robots, the actuator and energy extractor are separated. In living organisms, these components can be found in highly integrated systems. Energy transfer during the movement of animals does not have a clear mathematical mode. Many key techniques for fluid power systems in nature have attracted much interest, and the model uncertainty and parameter variations are of major value in natural designs. The muscle-fluid-cylinder compactness, integration, and weight systems of the hydrostatic skeleton are not well understood. An understanding of natural fluid power systems is needed to enable researchers to develop better, more accurate models. The goal of biorobotics is to take inspiration from these organisms to design robots using hydrodynamics, biomechanics, neuroscience, and prosthetics.

Biological hydraulics can provide support for the development of engineered hydraulic systems. Engineered hydraulic systems suffer from a low efficiency and can cause environmental damage; for example, using high-voltage and high-speed flow to improve power density generates problems with efficiency reduction and noise, as well as problems associated with the medium leakage and environmental pollution. These are all unwanted environmental disturbances. Similarly, living systems fit seamlessly with their environment, particularly when the body fluid or the external environment working medium is water, which is an environmentally-friendly, cheap, and inexhaustible medium. The organism itself is characterized by a light weight, high power density, and high reliability, characteristics that are the continuous pursuit of engineered hydraulic systems.

Soft-bodied robots and fluidic actuators are increasingly attracting attention. New soft robots can, in principle, be designed to provide outstanding mobility, strength, and reliability. Soft technologies will greatly assist in the development of robots capable of substantial interaction with an environment or human users by providing safer and more robust interactions than are currently available with conventional robotics, adaptive behaviors that use mechanical intelligence and thereby simplify the controller's needed for physical interaction, and cheaper and simpler robotic components than those currently available. There are many attractive avenues for the future work, 
development, and applications for this technology. With the rapid development of soft robots, intelligent drives with intelligent materials and combined 3D/4D technologies will allow for the advancement of their own control ability. Researchers must integrate biological environmental awareness, self-management and diagnosis, compliant drives, and self-adaptation to achieve intelligent systems.

One of the important development trends in hydraulic transmission systems is the integration of a hydraulic drive, and an intelligent and integrated drive system is a key component of high-end equipment. Biological hydraulic drives can also inform the development of hydraulic transmission systems. Living systems are highly integrated. The fluid-filled cavity skeleton is a highly integrated power generation and transmission system, and the body works as a pump and soft actuatedhydraulic cylinder through deformation. Biological systems are able to achieve the precise control of their own movement. Whether it involves a high speed or low speed, and regardless of the type of motion, the precise control of motion requires control of the fluid transmission process. Whether the detection and control mechanisms of the fluid state in the process of biological hydraulic transmission are consistent with digital hydraulics is an issue worth studying in depth.

\section{Acknowledgments}

This work was partly supported by the National Natural Science Foundation of China [grant number 51675219], the National Natural Science Foundation of China [grant number 91848204], the PreResearch Foundation of Equipment Development Department and Ministry of Education of China [grant number 6141A02022614], and the China Postdoctoral Science Foundation [grant number 2016M590261].

\section{References}

[1] Rabie M G. Fluid power engineering. 2009.

[2] Backe W. The present and future of fluid power. Proceedings of the Institution of Mechanical Engineers, Part I: Journal of Systems and Control Engineering, 1993, 207(4): 193212.

[3] Burrows C R. Fluid power systems - some research issues. Proceedings of the Institution of Mechanical Engineers, Part C: Journal of Mechanical Engineering Science, 2000, 214(1): 203-220.

[4] Yang H Y, Pan M. Engineering research in fluid power: a review. Journal of Zhejiang University-Science A, 2015, 16(6): 427-442.

[5] Edge K A. The control of fluid power systems-responding to the challenges. Proceedings of the Institution of Mechanical Engineers, Part I: Journal of Systems and Control Engineering, 1997, 211(2): 91-110.

[6] Pfeifer R, Lungarella M, Iida F. Self-organization, embodiment, and biologically inspired robotics. Science, 2007, 318(5853): 1088-1093.

[7] Parry D A, Brown R H J. The hydraulic mechanism of the spider leg. Journal of Experimental Biology, 1959, 36(2): 423-433.

[8] Prusch R D, Whoriskey F. Maintenance of fluid volume in the starfish water vascular system. Nature, 1976, 262(5569): 577-578.

[9] Cahn R W. Biomimetics: Biologically inspired technologies. Nature, 2006, 444(7118): 425-426. 
[10] Meller M, Garcia E. Bioinspired hydraulic control systems. Proc Spie, 2013, 8686(868603): 11 .

[11] Ugurlu B, Havoutis I, Semini C, Caldwell D G. Dynamic Trot-Walking with the Hydraulic Quadruped Robot - HyQ: Analytical Trajectory Generation and Active Compliance Control. 2013 Ieee/Rsj International Conference on Intelligent Robots and Systems (Iros), 2013, 60446051.

[12] Semini C, Tsagarakis N G, Guglielmino E, Focchi M, Cannella F, Caldwell D G. Design of HyQ - a hydraulically and electrically actuated quadruped robot. Proceedings of the Institution of Mechanical Engineers, Part I: Journal of Systems and Control Engineering, 2011, 225(6): 831-849.

[13] Semini C, Goldsmith J, Rehman B U, Frigerio M, Barasuol V, Focchi M. DESIGN OVERVIEW OF THE HYDRAULIC QUADRUPED ROBOTS HyQ2MAX AND HyQ2CENTAUR. The Fourteenth Scandinavian International Conference on Fluid Power, $2015,: 20-22$.

[14] Zhu J, Wang Y, Jiang J L, Sun B, Cao H. Unidirectional variable stiffness hydraulic actuator for load-carrying knee exoskeleton. International Journal of Advanced Robotic Systems, 2017, 14(1): 1-12.

[15] Aliman N, Ramli R, Haris S M. Design and development of lower limb exoskeletons: A survey. Robotics and Autonomous Systems, 2017, 95102-116.

[16] Whitman J S, Meller M, Garcia E. Improving energy efficiency in robot limbs through hydraulic dangle. Bioinspiration, Biomimetics, and Bioreplication International Society for Optics and Photonics, 2015, 9429;94291M.

[17] Moalli J E, Summers A P. The wonders of whirl. Nature, 2016, 538(7625): 315-315.

[18] Vogel S. Nature's pumps. American Scientist, 1994, 82: 464-471.

[19] Vogel S. Living in a physical world - X. Pumping fluids through conduits. Journal of Biosciences, 2007, 32(2): 207-222.

[20] Vogel S. Life in moving fluids: the physical biology of flow. Princeton University Press, 1994 ,

[21] Vogel S. Comparative biomechanics: life's physical world. Princeton University Press, 2013 ,

[22] Vogel S. Why the Wheel Is Round: Muscles, Technology, and How We Make Things Move. 2016.

[23] Bach D, Schmich F, Masselter T, Speck T. A review of selected pumping systems in nature and engineering--potential biomimetic concepts for improving displacement pumps and pulsation damping. Bioinspiration \& biomimetics, 2015, 10(5): 051001.

[24] Trimmer B. A Journal of Soft Robotics: Why Now? Soft robotics, 2014, 1(1): 1-4.

[25] Afolayan M O. Copying Nature - A Design of Hyper-Redundant Robot Joint/Support Based on Hydrostatic Skeleton. Lect Notes Artif Int, 2015, 9222: 50-63.

[26] Messner W C, Paik J, Shepherd R, Kim S, Trimmer B A. Energy for Biomimetic Robots: Challenges and Solutions. Soft robotics, 2014, 1(2): 106-109.

[27] Li S, Vogt D M, Rus D, Wood R J. Fluid-driven origami-inspired artificial muscles. Proceedings of the National Academy of Sciences of the United States of America, 2017, 114(50): 13132-13137.

[28] Kier W M. The diversity of hydrostatic skeletons. The Journal of experimental biology, 
2012, 215(Pt 8): 1247-1257.

[29] Kochova P, Tonar Z. Structural and Mechanical Properties of Gastropod Connective and Smooth Muscle Tissue. Experimental Mechanics, 2014, 54(5): 791-803.

[30] Seo E, Ohishi K, Maruyama T, Imaizumi-Ohashi Y, Murakami M, Seo Y. Magnetic resonance imaging analysis of water flow in the mantle cavity of live Mytilus galloprovincialis. The Journal of experimental biology, 2014, 217(Pt 13): 2277-2287.

[31] Szymik B G, Satterlie R A. Circulation of hemocoelic fluid during slow and fast swimming in the pteropod mollusc Clione limacina. Invertebrate Biology, 2017, 136(3): 290-300.

[32] Liu C B, Chen S S, Sheng C, Qian Z H, Ren L Q, Ren L. Study on Bionic Hydraulic Driving Mechanism of Spider and its Bio-inspiration. Journal of Jilin University(Engineering and Technology Edition), [2019-09-18], (http://www.cnki.net/): (in chinese) .

[33] Ijspeert A J. Biorobotics: using robots to emulate and investigate agile locomotion. Science, 2014, 346(6206): 196-203.

[34] Booster N A, Su F Y, Adolph S C, Ahn A N. Effect of temperature on leg kinematics in sprinting tarantulas (Aphonopelma hentzi): high speed may limit hydraulic joint actuation. The Journal of experimental biology, 2015, 218(Pt 7): 977-982.

[35] Fratzl P. Applied physics: The virtues of tiling. Nature, 2014, 516(7530): 178-179.

[36] Palmgren P. The mechanism of the extrinsic coxal muscles of spiders. Ann Zool Fennici, 1981, 18(4): 203-207.

[37] Kropf C. Hydraulic system of locomotion. 2013.

[38] Blickhan R, And Friedrich G. Barth. Strains in the exoskeleton of spiders Journal of Comparative Physiology A, 1985, 157(1): 115-147.

[39] Wirkner, Christian S., Stefan R. Evolutionary morphology of the circulatory system in Peracarida (Malacostraca; Crustacea). Cladistics, 2010, 26:143-167.

[40] Huckstorf K, Kosok G, Seyfarth E A, Wirkner C S. The hemolymph vascular system in Cupiennius salei (Araneae: Ctenidae). Zoologischer Anzeiger, 2013, 252(1): 76-87.

[41] Shultz J W. Muscular anatomy of the giant whipscorpion Mastigoproctus giganteus (Lucas)(Arachnida: Uropygi) and its evolutionary significance. Zoological Journal of the Linnean Society, 2010, 108(4): 335-365.

[42] Shultz J W. Evolution of locomotion in arachnida: The hydraulic pressure pump of the giant whipscorpion, Mastigoproctus Giganteus (Uropygi). Journal of morphology, 1991, 210(1): 13-31.

[43] Whitehead W F, And J. G. Rempel. A study of the musculature of the black widow spider, Latrodectus mactans (Fabr.). Canadian Journal of Zoology, 1959, 37(6): 831-870.

[44] Anderson J F, Prestwich K N. The fluid pressure pumps of spiders (Chelicerata, Araneae). Zeitschrift für Morphologie der Tiere, 1975, 81(4): 257-277.

[45] Wilson R S. Some comments on the hydrostatic system of spiders (Chelicerata, Araneae). Zeitschrift für Morphologie der Tiere, 1970, 68(4): 308-322.

[46] Paul R J, Bihlmayer S, Colmorgen M, Zahler S. The open circulatory system of spiders (Eurypelma californicum, Pholcus phalangioides): a survey of functional morphology and physiology. Physiological Zoology, 1994, 67(6): 1360-1382.

[47] Wirkner, Christian S., Katarina H. The circulatory system of spiders. Spider Ecophysiology: Springer Berlin Heidelberg, 2013.

[48] Wilson R S, And Bullock J. The hydraulic interaction between prosoma and opisthosoma 
in Amaurobius ferox (Chelicerata, Araneae). Zoomorphology, 1973, 74(3): 221-230.

[49] Geiler H, Beier R. Nachweis glatter Muskulatur im Opisthosoma von Araneus diadematus Clerck. Zoologischer Anzeiger 1971,

[50] Robinson G L, Paim U. Opisthosomal musculature of female Araneus diadematus (Araneae: Argiopidae). The Canadian Entomologist, 1969, 101(4): 16.

[51] Stewart D M, Arthur W M. Blood pressure in the tarantula, Dugesiella hentzi. Journal of Comparative Physiology A: Neuroethology, Sensory, Neural, and Behavioral Physiology, 1974, 88(2): 141-172.

[52] Nentwig W. The species referred to as Eurypelma californicum (Theraphosidae) in more than 100 publications is likely to be Aphonopelma hentzi. Journal of Arachnology, 2012, 40(1): 128-130.

[53] Paul R J, Bihlmayer S. CIRCULATORY PHYSIOLOGY OF A TARANTULA (EURYPELMA-CALIFORNICUM). ZOOLOGY-ANALYSIS OF COMPLEX SYSTEMS, 1994, 98(2): 69-81.

[54] Mcmahon B R, Louis E B. The crustacean open circulatory system:a reexamination. Physiological Zoology, 1990, 35-71.

[55] Barth F G. Spider mechanoreceptors. Current opinion in neurobiology, 2004, 14(4): 415422.

[56] Schaber C F, Gorb S N, Barth F G. Force transformation in spider strain sensors: white light interferometry. Journal of the Royal Society, Interface, 2012, 9(71): 1254-1264.

[57] Fratzl P, Barth F G. Biomaterial systems for mechanosensing and actuation. Nature, 2009, 462(7272): 442-448.

[58] Kang D, Pikhitsa P V, Choi Y W, Lee C, Shin S S, Piao L, Park B, Suh K Y, Kim T I, Choi M. Ultrasensitive mechanical crack-based sensor inspired by the spider sensory system. Nature, 2014, 516(7530): 222-226.

[59] Weihmann T, Gunther M, Blickhan R. Hydraulic leg extension is not necessarily the main drive in large spiders. The Journal of experimental biology, 2012, 215( $\mathrm{Pt} 4$ ): 578-583.

[60] Sensenig A T, Shultz J W. Mechanics of cuticular elastic energy storage in leg joints lacking extensor muscles in arachnids. The Journal of experimental biology, 2003, 206(Pt 4): 771-784. [61] Sensenig A T, Shultz J W. Elastic energy storage in the pedipalpal joints of scorpions and sun-spiders (Arachnida, Scorpiones, Solifugae). Journal of Arachnology, 2004, 32(1): 1-10.

[62] Rubin S, Young M H, Wright J C, Whitaker D L, Ahn A N. Exceptional running and turning performance in a mite. The Journal of experimental biology, 2016, 219(Pt 5): 676-685.

[63] Heethoff M, Koerner L. Small but powerful: the oribatid mite Archegozetes longisetosus Aoki (Acari, Oribatida) produces disproportionately high forces. The Journal of experimental biology, 2007, 210(Pt 17): 3036-3042.

[64] Schmelzle S, Norton R A, Heethoff M. Mechanics of the ptychoid defense mechanism in Ptyctima (Acari, Oribatida): One problem, two solutions. Zoologischer Anzeiger, 2015, 254:2740.

[65] Wauthy G, Leponce M, Banaï N, Sylin G, Lions J C. The backward jump of a box moss mite. Proceedings of the Royal Society of London Series B: Biological Sciences, 1998, 265(1411): 2235-2242.

[66] Miller G. Behavioral neuroscience uncaged. Science, 2004, 306(5695): 432-434.

[67] Politi Y, Priewasser M, Pippel E, Zaslansky P, Hartmann J, Siegel S, Li C H, Barth F G, 
Fratzl P. A Spider's Fang: How to Design an Injection Needle Using Chitin-Based Composite Material. Advanced Functional Materials, 2012, 22(12): 2519-2528.

[68] Siebert T, Weihmann T, Rode C, Blickhan R. Cupiennius salei: biomechanical properties of the tibia-metatarsus joint and its flexing muscles. Journal of comparative physiology B, 2010, 180(2): 199-209.

[69] Weihmann T. Crawling at High Speeds: Steady Level Locomotion in the Spider Cupiennius salei-Global Kinematics and Implications for Centre of Mass Dynamics. PloS one, 2013, 8(6): e65788.

[70] Dippenaarschoeman A S, Jocque R. African spiders: an identification manual. 1997.

[71] Wood H M, Parkinson D Y, Griswold C E, Gillespie R G, Elias D O. Repeated Evolution of Power-Amplified Predatory Strikes in Trap-Jaw Spiders. Current biology : CB, 2016, 26(8): 1057-1061.

[72] Théry, Marc, Casas, Jér?Me. Predator and prey views of spider camouflage. Nature, 2002, 415(6868): 133.

[73] Tanaka Y, Hisada M. The hydraulic mechanism of the predatory strike in dragonfly larvae[J]. Journal of experimental biology. Journal of experimental biology, 1980, 88(88): 119.

[74] Casey T M. Energetics of caterpillar locomotion: biomechanical constraints of a hydraulic skeleton. Science, 1991, 252(5002): 112-114.

[75] Simon M A, Woods W A, Jr., Serebrenik Y V, Simon S M, Van Griethuijsen L I, Socha J J, Lee W K, Trimmer B A. Visceral-locomotory pistoning in crawling caterpillars. Current biology : $C B, 2010,20(16):$ 1458-1463.

[76] Brackenbury J. Fast locomotion in caterpillars. Journal of insect physiology, 1999, 45(6): 525-533.

[77] Trueman E R, Brown A C. Locomotion, pedal retraction and extension, and the hydraulic systems of Bullia (Gastropoda: Nassaridae). Journal of Zoology 1976, 178(3): 365-384.

[78] Dale B. Blood pressure and its hydraulic functions in Helix pomatia L. Journal of Experimental Biology, 1973, 59(2): 477-490.

[79] Dorgan K M, D'amelio C, Lindsay S M. Strategies of burrowing in soft muddy sediments by diverse polychaetes. Invertebrate Biology, 2016, 135(4): 287-301.

[80] Che J, Dorgan K M. Mechanics and kinematics of backward burrowing by the polychaete Cirriformia moorei. The Journal of experimental biology, 2010, 213(Pt 24): 4272-4277.

[81] Vincent J F V. Deployable structures in nature: potential for biomimicking. 2000.

[82] Satterlie N R A. Co-activation of antagonistic motoneurons as a mechanism of highspeed hydraulic inflation of prey capture appendages in the pteropod mollusk Clione limacina. The Biological bulletin, 1993, 185(2): 240-247.

[83] Salisbury S M, Martin G G, Kier W M, Schulz J R. Venom kinematics during prey capture in Conus: the biomechanics of a rapid injection system. The Journal of experimental biology, 2010, 213(5): 673-682.

[84] Triantafyllou M S, Weymouth G D, Miao J M. Biomimetic Survival Hydrodynamics and Flow Sensing. Annu Rev Fluid Mech, 2016, 48(1): 1-24.

[85] Zeidberg L D, Robison B H. Invasive range expansion by the Humboldt squid, Dosidicus gigas, in the eastern North Pacific. Proceedings of the National Academy of Sciences of the United States of America, 2007, 104(31): 12948-12950. 
[86] Rosenbluth J, Szent-Gyorgyi A G, Thompson J T. The ultrastructure and contractile properties of a fast-acting, obliquely striated, myosin-regulated muscle: the funnel retractor of squids. The Journal of experimental biology, 2010, 213(Pt 14): 2430-2443.

[87] Anderson E J, Grosenbaugh M A. Jet flow in steadily swimming adult squid. The Journal of experimental biology, 2005, 208(Pt 6): 1125-1146.

[88] Bartol I K, Krueger P S, Thompson J T, Stewart W J. Swimming dynamics and propulsive efficiency of squids throughout ontogeny. Integrative and comparative biology, 2008, 48(6): 720-733.

[89] Thompson J T, Kier W M. Ontogeny of mantle musculature and implications for jet locomotion in oval squid Sepioteuthis lessoniana. The Journal of experimental biology, 2006, 209(Pt 3): 433-443.

[90] Staaf D J, Gilly W F, Denny M W. Aperture effects in squid jet propulsion. The Journal of experimental biology, 2014, 217(Pt 9): 1588-1600.

[91] Cox K. The Unique Ecology of Lembeh Strait, Indonesia. Fisheries, 2017, 42(10): 519525.

[92] Woodin W S A. Infaunal hydraulics generate porewater pressure signals. The Biological bulletin, 2005, 209(2): 139-145.

[93] Trueman E R. The dynamics of burrowing in Ensis (Bibalvia). Proceedings of the Royal Society of London Series B, Biological sciences, 1967, 166(1005): 459-476.

[94] Trueman E R, Brown A C. Dynamics of burrowing and pedal extension in Donax serra (Mollusca: Bivalvia). Journal of Zoology, 1985, 207(3): 345-355.

[95]Ansell A D, Trueman E R. Burrowing in Mercenaria mercenaria (L.)(Bivalvia, Veneridae). . Journal of Experimental Biology, 1967, 46(1): 105-115.

[96] Dorgan K M. The biomechanics of burrowing and boring. The Journal of experimental biology, 2015, 218(Pt 2): 176-183.

[97] B.A. Skierczynski, Wilson R J A, Jr W B K, Skalak R. A model of the hydrostatic skeleton of the leech. Journal of Theoretical Biology, 1996, 181(4): 329-342.

[98] Vaidyanathan R, Chiel H J, Quinn R D. A hydrostatic robot for marine applications. Robotics and Autonomous Systems, 2000, 30(1-2): 103-113.

[99] Dorgan K M. Environmental Constraints on the Mechanics of Crawling and Burrowing Using Hydrostatic Skeletons. Experimental Mechanics, 2010, 50(9): 1373-1381.

[100] Trimmer B A, Lin H T. Bone-free: soft mechanics for adaptive locomotion. Integrative and comparative biology, 2014, 54(6): 1122-1135.

[101] Sturzenbaum S R, Hockner M, Panneerselvam A, Levitt J, Bouillard J S, Taniguchi S, Dailey L A, Ahmad Khanbeigi R, Rosca E V, Thanou M, Suhling K, Zayats A V, Green M. Biosynthesis of luminescent quantum dots in an earthworm. Nature nanotechnology, 2013, 8(1): 57-60.

[102] Quillin K J. Ontogenetic scaling of hydrostatic skeletons: geometric, static stress and dynamic stress scaling of the earthworm lumbricus terrestris. The Journal of experimental biology, 1998, 201 (Pt 12)1871-1883.

[103] Quillin K J. Kinematic scaling of locomotion by hydrostatic animals: ontogeny of peristaltic crawling by the earthworm lumbricus terrestris. The Journal of experimental biology, 1999, 202 (Pt 6)661-674.

[104] Yekutieli Y, Flash T, Hochner B. Biomechanics: Hydroskeletal. 2009, 189-200. 
[105] Hamilton G. The secret lives of jellyfish. Nature, 2016, 531(7595): 432-434.

[106] Baumgarten S, Simakov O, Esherick L Y, Liew Y J, Lehnert E M, Michell C T, Li Y, Hambleton E A, Guse A, Oates M E, Gough J, Weis V M, Aranda M, Pringle J R, Voolstra C R. The genome of Aiptasia, a sea anemone model for coral symbiosis. Proceedings of the National Academy of Sciences of the United States of America, 2015, 112(38): 11893-11898.

[107] Nawroth J C, Lee H, Feinberg A W, Ripplinger C M, Mccain M L, Grosberg A, Dabiri J O, Parker K K. A tissue-engineered jellyfish with biomimetic propulsion. Nature biotechnology, 2012, 30(8): 792-797.

[108] Gemmell B J, Colin S P, Costello J H, Dabiri J O. Suction-based propulsion as a basis for efficient animal swimming. Nature communications, 2015, 6:87-90.

[109] Park S G, Chang C B, Huang W X, Sung H J. Simulation of swimming oblate jellyfish with a paddling-based locomotion. Journal of Fluid Mechanics, 2014, 748:731-755.

[110] Gemmell B J, Costello J H, Colin S P, Stewart C J, Dabiri J O, Tafti D, Priya S. Passive energy recapture in jellyfish contributes to propulsive advantage over other metazoans. Proceedings of the National Academy of Sciences of the United States of America, 2013, 110(44): 17904-17909.

[111] Park S G, Kim B, Lee J, Huang W X, Sung H J. Dynamics of prolate jellyfish with a jet-based locomotion. Journal of Fluids and Structures, 2015, 57331-343.

[112] Santos R, Haesaerts D, Jangoux M, Flammang P. Comparative histological and immunohistochemical study of sea star tube feet (Echinodermata, Asteroidea). Journal of morphology, 2005, 263(3): 259-269.

[113] Hennebert E. Adhesion mechanisms developed by sea stars: A review of the ultrastructure and composition of tube feet and their secretion. Biological Adhesive Systems.: Springer Vienna, 2010.

[114] Skyler R, Kier W M. The Functional Morphology of Starfish Tube Feet: The Role of a Crossed-Fiber Helical Array in Movement. The Biological bulletin, 1995, 188(2): 197-209.

[115] Santos R, Gorb S, Jamar V, Flammang P. Adhesion of echinoderm tube feet to rough surfaces. The Journal of experimental biology, 2005, 208(Pt 13): 2555-2567.

[116] Hennebert E, Haesaerts D, Dubois P, Flammang P. Evaluation of the different forces brought into play during tube foot activities in sea stars. The Journal of experimental biology, 2010, 213(Pt 7): 1162-1174.

[117] Santos R, Barreto A, Franco C, Coelho A V. Mapping sea urchins tube feet proteome-a unique hydraulic mechano-sensory adhesive organ. Journal of proteomics, 2013, 79100-113. [118] Bone Q, Trueman E R. Jet propulsion in salps. Journal of Zoology, 1983, 201(4): 481506.

[119] Sutherland K R, Madin L P. Comparative jet wake structure and swimming performance of salps. The Journal of experimental biology, 2010, 213(Pt 17): 2967-2975.

[120] Madin L P. Aspects of jet propulsion in salps. Canadian Journal of Zoology, 1990, 68(4): $765-777$.

[121] Polygerinos P, Correll N, Morin S A, Mosadegh B, Onal C D, Petersen K, Cianchetti M, Tolley M T, Shepherd R F. Soft Robotics: Review of Fluid-Driven Intrinsically Soft Devices; Manufacturing, Sensing, Control, and Applications in Human-Robot Interaction Advanced Engineering Materials, 2017, 19(12): 1700016.

[122] Wehner M, Truby R L, Fitzgerald D J, Mosadegh B, Whitesides G M, Lewis J A, 
Wood R J. An integrated design and fabrication strategy for entirely soft, autonomous robots. Nature, 2016, 536(7617): 451-455.

[123] Hines L, Petersen K, Lum G Z, Sitti M. Soft Actuators for Small-Scale Robotics. Advanced materials, 2017, 29(13): 1603483.

[124] Bryant M, Meller M A, Garcia E. Variable recruitment fluidic artificial muscles: modeling and experiments. Smart Materials and Structures, 2014, 23(7): 074009.

[125] Desbiens a B , Lucking Bigué, Jean-Philippe, Véronneau C, Masson P, Iagnemma K, Plante J S. On the Potential of Hydrogen-Powered Hydraulic Pumps for Soft Robotics. Soft robotics, 2017, :soro.2016.0071.

[126] Hines L, Petersen K, Sitti M. Inflated Soft Actuators with Reversible Stable Deformations. Advanced materials, 2016, 28(19): 3690-3696.

[127] Katzschmann R K, De Maille A, Dorhout D L, Rus D. Cyclic Hydraulic Actuation for Soft Robotic Devices. 2016 Ieee/Rsj International Conference on Intelligent Robots and Systems (Iros 2016), 2016, 3048-3055.

[128] Tiwari R, Meller M A, Wajcs K B, Moses C, Reveles I, Garcia E. Hydraulic artificial muscles. Journal of Intelligent Material Systems and Structures, 2012, 23(3): 301-312.

[129] Mirvakili S M, Hunter I W. Artificial Muscles: Mechanisms, Applications, and Challenges. Advanced materials, 2017, :1704407.

[130] Sridar S, Majeika C J, Schaffer P, Bowers M, Ueda S, Barth A J, Sorrells J L, Wu J T, Hunt T R, Popovic M. Hydro Muscle-A Novel Soft Fluidic Actuator. 2016 Ieee International Conference on Robotics and Automation (Icra), 2016, 4014-4021.

[131] Farrow N, Correll N. A Soft Pneumatic Actuator that Can Sense Grasp and Touch. Ieee Int C Int Robot, 2015, 2317-2323.

[132] Polygerinos P, Wang Z, Overvelde J T B, Galloway K C, Wood R J, Bertoldi K, Walsh C J. Modeling of Soft Fiber-Reinforced Bending Actuators. Ieee T Robot, 2015, 31(3): 778-789.

[133] Wang H, Ma J G, Ren Z Y, Gong Z Y, Hao Y F, Wang T M, Wen L. Fiber-reinforced Soft Robotic Anthropomorphic Finger. 2016 International Conference on Robotics and Automation Engineering (ICRAE 2016), 2016, 1-5.

[134] Wirekoh J, Park Y L. Design of flat pneumatic artificial muscles. Smart Materials and Structures, 2017, 26(3): 035009.

[135] Robertson M A, Sadeghi H, Florez J M, Paik J. Soft Pneumatic Actuator Fascicles for High Force and Reliability. Soft robotics, 2017, 4(1): 23-32.

[136] Connolly F, Polygerinos P, Walsh C J, Bertoldi K. Mechanical Programming of Soft Actuators by Varying Fiber Angle. Soft robotics, 2015, 2(1): 26-32.

[137] Al-Fahaam H, Davis S, Nefti-Meziani S. The design and mathematical modelling of novel extensor bending pneumatic artificial muscles (EBPAMs) for soft exoskeletons. Robotics and Autonomous Systems, 2018, 9963-74.

[138] Tarvainen T V J, Yu W W. Pneumatic Multi-Pocket Elastomer Actuators for Metacarpophalangeal Joint Flexion and Abduction-Adduction. Actuators, 2017, 6(3): 27.

[139] Bishop-Moser J, Kota S. Design and Modeling of Generalized Fiber-Reinforced Pneumatic Soft Actuators. Ieee T Robot, 2015, 31(3): 536-545.

[140] Onal C D, Chen X, Whitesides G M, Rus D. Soft Mobile Robots with On-Board Chemical Pressure Generation. Springer Trac Adv Ro, 2017, 100 
[141] Gamus B, Salem L, Ben-Haim E, Gat A D, Or Y. Interaction Between Inertia, Viscosity, and Elasticity in Soft Robotic Actuator With Fluidic Network. Ieee T Robot, 2018, 34(1): 81-90.

[142] Kelly D A. Penises as variable-volume hydrostatic skeletons. Annals of the New York Academy of Sciences, 2007, 1101(1): 453-463.

[143] Loepfe M, Schumacher C M, Burri C H, Stark W J. Contrast Agent Incorporation into Silicone Enables Real-Time Flow-Structure Analysis of Mammalian Vein-Inspired Soft Pumps. Advanced Functional Materials, 2015, 25(14): 2129-2137.

[144] Roche E T, Wohlfarth R, Overvelde J T, Vasilyev N V, Pigula F A, Mooney D J, Bertoldi K, Walsh C J. A bioinspired soft actuated material. Advanced materials, 2014, 26(8): 1200-1206.

[145] Wu W, Deconinck A, Lewis J A. Omnidirectional printing of 3D microvascular networks. Advanced materials, 2011, 23(24): 0-0.

[146] Kolesky D B, Truby R L, Gladman A S, Busbee T A, Homan K A, Lewis J A. 3D bioprinting of vascularized, heterogeneous cell-laden tissue constructs. Advanced materials, 2014, 26(19): 3124-3130.

[147] Wegst U G, Bai H, Saiz E, Tomsia A P, Ritchie R O. Bioinspired structural materials. Nature materials, 2015, 14(1): 23-36.

[148] Ortiz C, Boyce M C. Materials science. Bioinspired structural materials. Science, 2008, 319(5866): 1053-1054.

[149] Acome E, Mitchell S K, Morrissey T G, Emmett M B, Benjamin C, King M, Radakovitz M, Keplinger C. Hydraulically amplified self-healing electrostatic actuators with muscle-like performance. Science, 2018, 359(6371): 61-65.

[150] Yuk H, Lin S, Ma C, Takaffoli M, Fang N X, Zhao X. Hydraulic hydrogel actuators and robots optically and sonically camouflaged in water. Nature communications, 2017, 8: 14230 .

[151] Gerboni G, Diodato A, Ciuti G, Cianchetti M, Menciassi A. Feedback Control of Soft Robot Actuators via Commercial Flex Bend Sensors. Ieee-Asme T Mech, 2017, 22(4): 18811888.

[152] Li S, Zhao H C, Shepherd R F. Flexible and stretchable sensors for fluidic elastomer actuated soft robots. Mrs Bulletin, 2017, 42(2): 138-142.

[153] Han D, Gu H R, Kim J W, Yokota S. A bio-inspired 3D-printed hybrid finger with integrated ECF (electro-conjugate fluid) micropumps. Sensor Actuat a-Phys, 2017, 25747-57.

[154] Felt W, Chin K Y, Remy C D. Contraction Sensing with Smart Braid McKibben Muscles. IEEE/ASME transactions on mechatronics : a joint publication of the IEEE Industrial Electronics Society and the ASME Dynamic Systems and Control Division, 2016, 21(3): 12011209.

[155] Elgeneidy K, Lohse N, Jackson M. Bending angle prediction and control of soft pneumatic actuators with embedded flex sensors - A data-driven approach. Mechatronics, 2018, 50:234-247.

[156] Gladman A S, Matsumoto E A, Nuzzo R G, Mahadevan L, Lewis J A. Biomimetic 4D printing. Nature materials, 2016, 15(4): 413-418.

[157] Khoo Z X, Teoh J E M, Liu Y, Chua C K, Yang S F, An J, Leong K F, Yeong W Y. 3D printing of smart materials: A review on recent progresses in 4D printing. Virtual and Physical 
Prototyping, 2015, 10(3): 103-122.

[158] Murphy S V, Atala A. 3D bioprinting of tissues and organs. Nature biotechnology, 2014, 32(8): 773-785.

[159] Niiyama R, Sun X, Sung C, An B, Rus D, Kim S. Pouch Motors: Printable Soft Actuators Integrated with Computational Design. Soft robotics, 2015, 2(2): 59-70.

[160] Robinson S S, O'brien K W, Zhao H C, Peele B N, Larson C M, Mac Murray B C, Van Meerbeek I M, Dunham S N, Shepherd R F. Integrated soft sensors and elastomeric actuators for tactile machines with kinesthetic sense. Extreme Mechanics Letters, 2015, 547-53.

[161] Miriyev A, Stack K, Lipson H. Soft material for soft actuators. Nature communications, 2017, 8(1): 596.

[162] Yang D, Verma M S, So J-H, Mosadegh B, Keplinger C, Lee B, Khashai F, Lossner E, Suo Z, Whitesides G M. Buckling Pneumatic Linear Actuators Inspired by Muscle. Advanced Materials Technologies, 2016, 1(3): 1600055.

[163] Kang T Y, Kaminaga H, Nakamura Y. A Robot Hand Driven By Hydraulic Cluster Actuators. Ieee-Ras Int C Human, 2014, 39-44.

[164] Vincent J F V. Stealing Ideas from Nature. Deployable structures Springer Vienna, 2001, 51-58.

[165] Hu N, Feng P, Dai G L. The Gift from Nature: Bio-Inspired Strategy for Developing Innovative Bridges. Journal of Bionic Engineering, 2013, 10(4): 405-414.

[166] Ouyang X P, Ding S, Fan B Q, Li P Y, Yang H Y. Development of a novel compact hydraulic power unit for the exoskeleton robot. Mechatronics, 2016, 38:68-75.

[167] Maccurdy R, Katzschmann R, Kim Y, Rus D. Printable Hydraulics: A Method for Fabricating Robots by 3D Co-Printing Solids and Liquids. 2016 Ieee International Conference on Robotics and Automation (Icra), 2016, 3878-3885.

[168] Berring J, Kianfar K, Lira C, Menon C, Scarpa F. A smart hydraulic joint for future implementation in robotic structures. Robotica, 2010, 28(07): 1045-1056.

[169] Lira C, Menon C, Kianfar K, Scarpa F, Mani M. Mining Smartness from the Hydraulic System of Spiders: A Bioinspired Actuator for Advanced Applications. Advances in Science and Technology, 2008, 58:114-119.

[170] Menon C, Lira C. Active articulation for future space applications inspired by the hydraulic system of spiders. Bioinspiration \& biomimetics, 2006, 1(2): 52-61.

[171] Schulz S, Pylatiuk C, Reischl M, Martin J, Mikut R, Bretthauer G. A hydraulically driven multifunctional prosthetic hand. Robotica, 2005, 23(3): 293-299.

[172] Landkammer S, Schneider D, Winter F, Hess P, Hornfeck R. Static Modeling of an Antagonistic Pneumatic Actuator for Robotic Applications. 2015 Ieee International Workshop of Electronics, Control, Measurement, Signals and Their Application to Mechatronics (Ecmsm), 2015, 1-6.

[173] Nemiroski A, Shevchenko Y Y, Stokes A A, Unal B, Ainla A, Albert S, Compton G, Macdonald E, Schwab Y, Zellhofer C, Whitesides G M. Arthrobots. Soft robotics, 2017, 4(3): 183-190.

[174] Berlin, Heidelberg. Encyclopedia of Neuroscience. Springer, 2009.

[175] Giorgio-Serchi F, Arienti A, Laschi C. Underwater soft-bodied pulsed-jet thrusters: Actuator modeling and performance profiling. Int J Robot Res, 2016, 35(11): 1395-1416. 